\title{
Agent Services for Situation Aware Control of Power Systems With Distributed Generation
}

Saleem, Arshad; Heussen, Kai; Lind, Morten

Published in:

IEEE Power \& Energy Society General Meeting, 2009. PES '09.

Link to article, DOI:

10.1109/PES.2009.5260219

Publication date:

2009

Document Version

Publisher's PDF, also known as Version of record

Link back to DTU Orbit

Citation (APA):

Saleem, A., Heussen, K., \& Lind, M. (2009). Agent Services for Situation Aware Control of Power Systems With Distributed Generation. In IEEE Power \& Energy Society General Meeting, 2009. PES '09.: IEEE PES General Meeting (GM) (1 ed.). IEEE. https://doi.org/10.1109/PES.2009.5260219

\section{General rights}

Copyright and moral rights for the publications made accessible in the public portal are retained by the authors and/or other copyright owners and it is a condition of accessing publications that users recognise and abide by the legal requirements associated with these rights.

- Users may download and print one copy of any publication from the public portal for the purpose of private study or research.

- You may not further distribute the material or use it for any profit-making activity or commercial gain

- You may freely distribute the URL identifying the publication in the public portal 


\title{
Agent Services for Situation Aware Control of Power Systems With Distributed Generation
}

\author{
Arshad Saleem, Student-Member, IEEE, Kai Heussen, Student-Member, IEEE, and Morten Lind
}

\begin{abstract}
Electric Power system of Denmark exhibits some unique characteristics. An increasing part of the electricity is produced by distributed generators (DGs). Most of these DGs are connected to the network at the distribution level. At the same time the concept of vehicle to grid (V2G) is already in the process of realization. This situation has created an incentive in electric power industry to utilize modern information and communication technologies (ICT) for improving the distribution system automation. This paper describes our work on how significantly increased amount of distributed generation could be exploited for the robust control of electric power systems. In particular, we present our work on the implementation of a dynamic service oriented system, in which autonomous agents represent different components of low voltage grid. These agents could offer and utilize electric power control services. We present results from several experiments where agents offer and utilize services in order to achieve distributed and autonomous control for subgrid operation of a distribution system. Finally it is discussed how the service oriented architecture can be combined with knowledge based reasoning to implement situation awareness required in complex control situations.
\end{abstract}

Index Terms-Intelligent Control, Smart Grid, Autonomous Agents, Services, Situation Awareness

\section{INTRODUCTION}

D ISTRIBUTED generation, decentralized and local control, self organization and autonomy are evident trends of future's electric power systems focusing on innovative control architectures like MicroGrids, virtual power plants, cell based systems and grid connected electric vehicles.

Realization of these concepts require that power systems should be of distributed nature - consisting of autonomous components - which are able to coordinate, communicate, compete, adapt to emerging situations and self organize themselves. Intelligent Software Agents which are autonomous software entities posses most of these capabilities in their design metaphor and have already proved a potential for providing such capabilities in other fields e.g. e-commerce. This fact has made agents a very interesting technology for design, control and operations of the smart electric power systems - the smart grid.

This paper describes our work on how significantly increased amount of distributed generation could be exploited for the control of electric power systems. In particular, we present our work for the implementation of a dynamic service oriented system. In this system autonomous agents represent different components of low voltage distribution grid, and both offer and utilize electric power control services. We present results from

All authors are affiliated with Centre for Electric Technology, Department of Electical Engineering, Technical Univeristy of Denmark, 2800 Kgs. Lyngby, Denmark

e-mail: \{asa,kh,mli\}@elektro.dtu.dk several experiments on distributed and autonomous control in subgrid operation where a part of the distribution network is islanded form the utility grid. We also motivate a mechanism for situation awareness in agents. It is described why it is critical for agents to be aware of the semantics of situation before being able to request,invoke or provide control services in complex situations.

Intelligent systems and autonomous agents have been of great interest for the control of electric power systems in recent years. Jennings [1] portrays a general case for using agent in control systems. In [2] a multiagent approach has been presented for restoration of electric power distribution networks with single source of power. It motivates the use of agents at different control levels e.g. feeder agents and load agents. This approach brings the ideas of hierarchal and distributed control together. McArthur et al. [3] suggests the use of agents in a services oriented manner for development of electric power engineering applications specially in the context of a shift from energy as product to energy supply as a service. Solanki et al. [4] presents a multi agent mechanism for islanding operation of distribution systems with DGs. It suggests an under voltage load shedding schema in the electrical islands for maintenance of energy balance.

In this paper we present a fully decentralized and service oriented approach where autonomous agents represent physical electric power system components like distributed generators (DG agents), electric power loads (Load agents ) and a load shedding agent (LS agents). In our approach DG agents register regulation services, and load agents register shedding services for the provision of power balance. Load shedding is performed when not enough regulation services are available to restore energy balance in the islanded subgrid. For load shedding, we adopt the idea of performing under voltage load shedding [4] because voltage drop is more severe then frequency drop in islanding scenarios of low voltage grid.

An important aspect of our approach is that both the provision of non-scheduled power from DGs for power balance, and the load shedding, is performed in a service oriented manner. DG agents choose to provide service of regulation, and load agent choose to shutdown themselves autonomously as a provision of service. This is critical for the realization of innovative architectures like microgrids and virtual power plants, where electric power components are owned by different owners with specific economic interest, and which also requires plug and play capabilities.

The present paper is organized as follows. Section II describes a dynamic model of low voltage grid which has been used in simulations. Section III describes a software platform developed for the implementation of autonomous agents and 


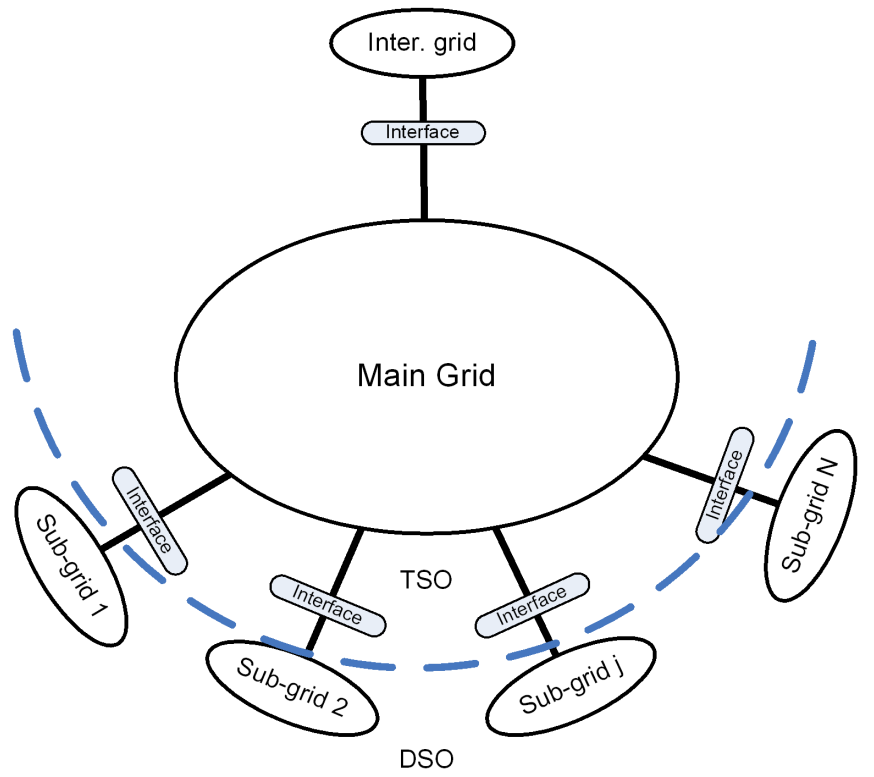

Fig. 1. Increased penetration of DGs in (Danish) electric power systems provides opportunity to control the electric power network as a subgrid based system, e.g. during islandng scenario [5], [6]

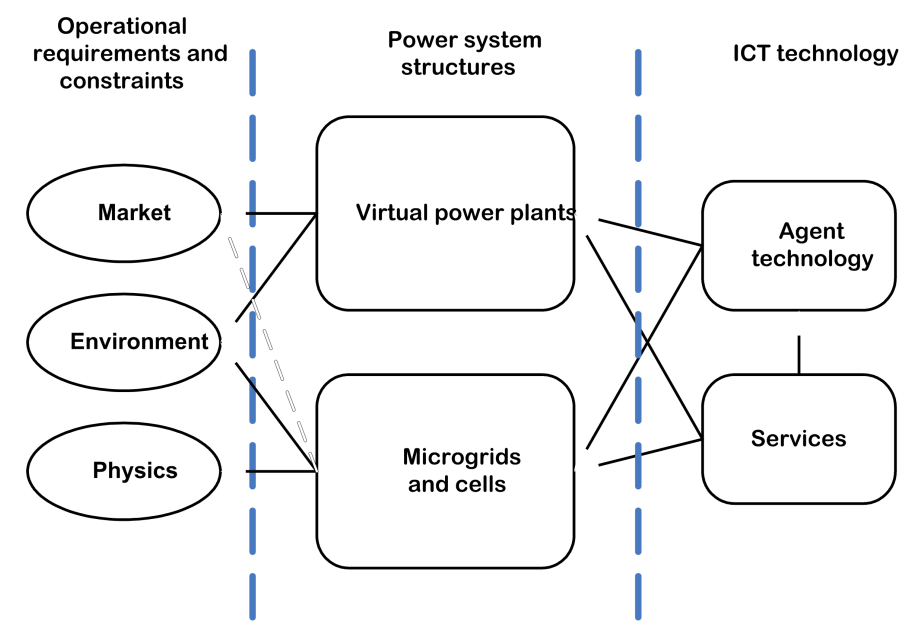

Fig. 2. Agents and service oriented technology can be used to implemen intelligence and autonomy required for the realization of innovative architectures like MicroGrid and Virtual Power Plants [5], [6]

services. It also describes development of a middleware for real time communication between the software platform implemented in JAVA and dynamic simulation model developed in DigSILENT PowerFactory ${ }^{1}$. Section IV describes simulations and results. Section V discusses how the service oriented architecture can be used to realize control agents which are situation aware. Section VI is conclusion section.

\section{Network MODELING}

A dynamic model of a part of the distribution grid has been modeled in DigSILENT PowerFactory for simulation and experiments purpose. Figure 3 shows a single line diagram of the model. It consists of three local DGs and four

\footnotetext{
${ }^{1}$ DIgSILENT GmbH: http://www.digsilent.de/
}

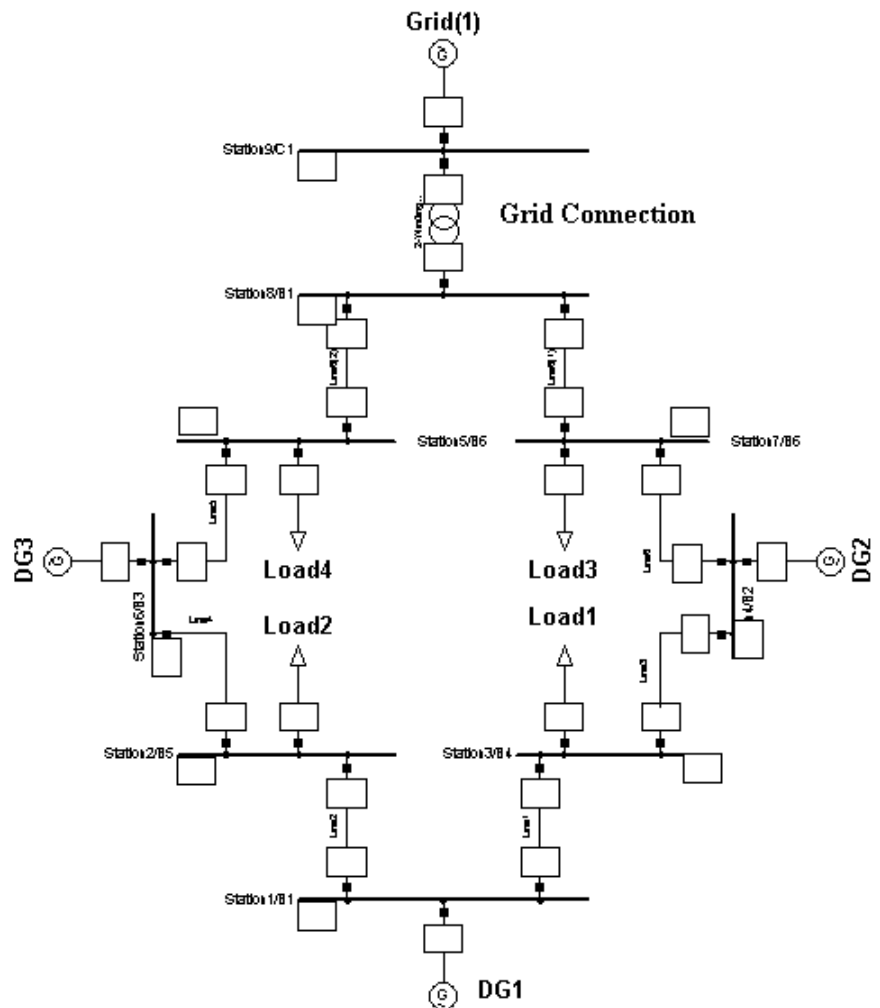

Fig. 3. Single line diagram of test model developed in DigSILENT PowerFactorey

(aggregated)loads. DGs are modeled using built-in type "4.9 MVA DG" of DigSILENT PowerFactory. A connection to the utility grid has also been simulated in the model. Initially three different scenarios have been simulated based upon this network model.

\section{Software Platform And Middleware DEVELOPMENT}

A dynamic multi-agent platform has been implemented in the Java agent development framework (JADE) ${ }^{2}$. The platform consists of one main container, and several sub containers. Each sub container represents an island in a distribution network consisting of one load shedding agent LS and several DG and load agents. Both DG and load agents can join or leave the network dynamically according to the changes in the network. New islands can also be created following any situation in the network. The software platform also includes JADE utility agents and services. Some of the important utilities and agents are: i) DF (directory facilitator) agent which provides yellow page services. DG and load agents interact with this agent to register and discover agent services, ii) AMS(agent management services)agent which provide white page services. This agent is responsible for creating, destroying and managing agents and containers in a JADE platform, iii) MTS (message transport service) is a service responsible for message transportation between agents with in a container and across containers. This service also

${ }^{2}$ Java Agent Development Framework (JADE): http://jade.tilab.com/ 


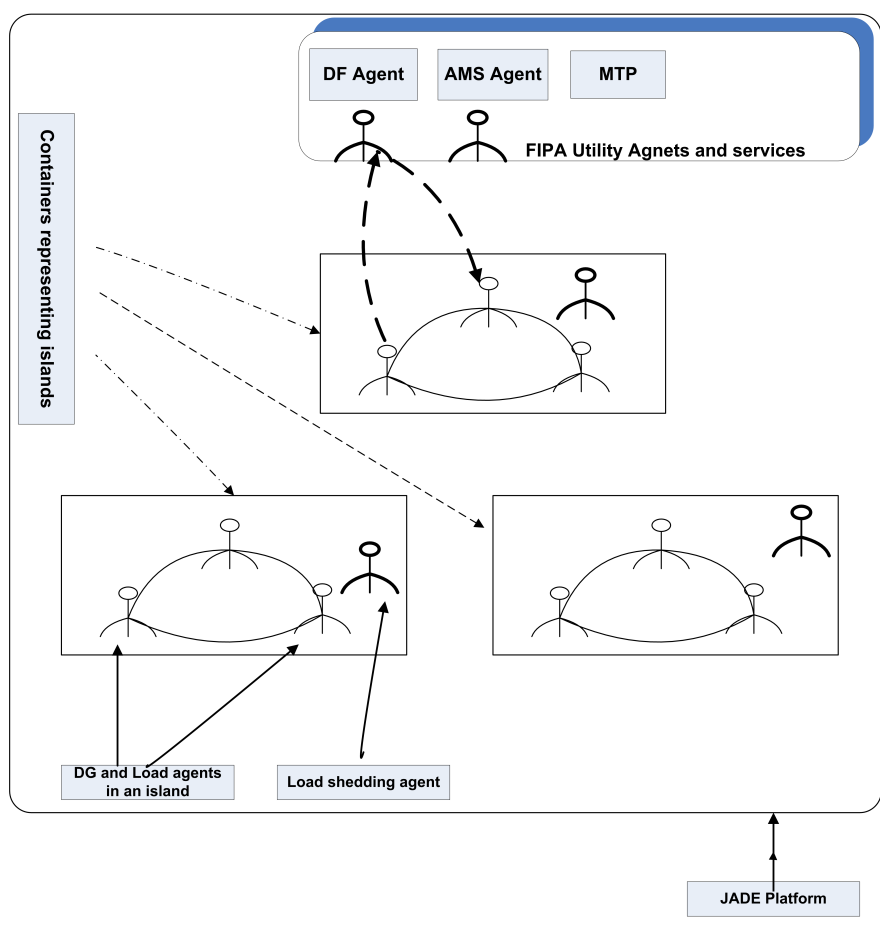

Fig. 4. Software platform developed in JADE

enables synchronization of messages when several messages are sent and received from different agents in parallel. In order to take full advantage of agent capabilities like autonomy, local control, scalability and high level communication, the software platform is implemented as fully compliant with FIPA (foundation for physical intelligent agents) standards ${ }^{3}$. Figure 4 shows the structure of the software platform. It presents a symbolic representation of containers which represent electrical islands in a distribution system, the agents inside these containers and the JADE utility services described above.

\section{A. Development of middleware software for communication between JADE and DigSILENT model}

Real time communication between the software platform and a dynamic simulation of physical network in "DigSILENT Power Factory" is implemented using a middleware based upon OPC (open connectivity via open standards) DA(real time data access) standard ${ }^{4}$. This middleware is implemented using java native interface (JNI) and fully conforms to the OPC standard. Through an OPC server, software agents can connect to respective devices in dynamic model and perform control actions during the simulations. Each agent in the software platform creates its own instance of connection and has an individual channel of control commands, which ensures that decentralized nature and robustness of the control mechanism is not compromised. Figure 5 shows structure of this middleware.

${ }^{3}$ Foundation for physical Intelligent Agents (FIPA):http://www.fipa.org/ ${ }^{4}$ OPC Foundation:http://www.opcfoundation.org/

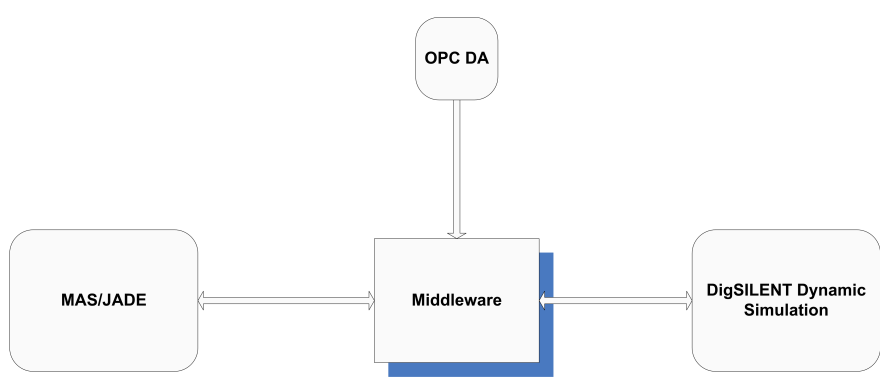

Fig. 5. Middleware development for realtime communication between dynamic simulation in Digsilent PowerFactorey and software platform

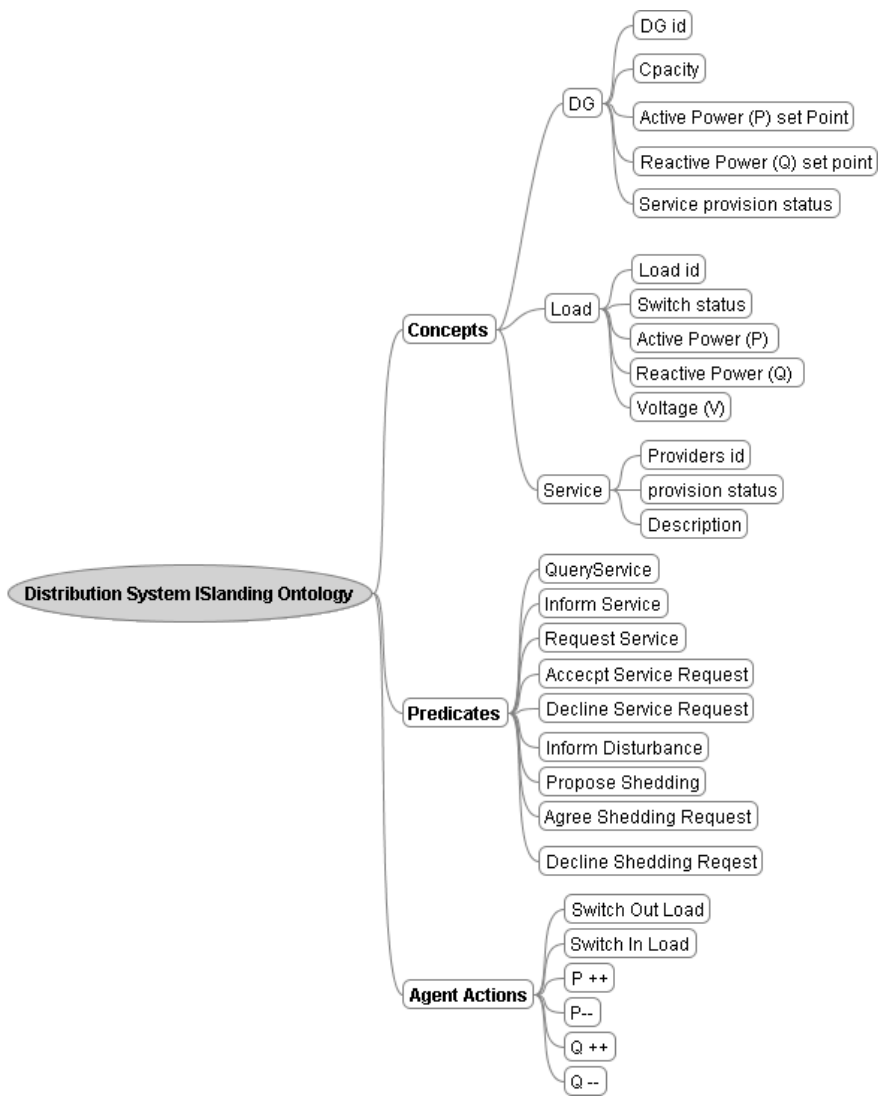

Fig. 6. Structure of ontology developed for agent knowledge realization and communication

\section{B. Development of Ontology for agent Communication}

Ontologies provide a way to structure information for several agents to understand the semantics of knowledge and to agree upon the terminologies used in communication. An ontology has been created to structure information transferred between agents. Basic components of this ontology are concepts, predicates and agent actions. The structure of this ontology called Distribution System Islanding Ontology is shown in figure 6. During the development of this ontology it has been made sure that it fully conforms to FIPA and W3C standards ${ }^{5}$.

${ }^{5}$ W3C - The World Wide Web Consortium :http://www.w3.org/ 

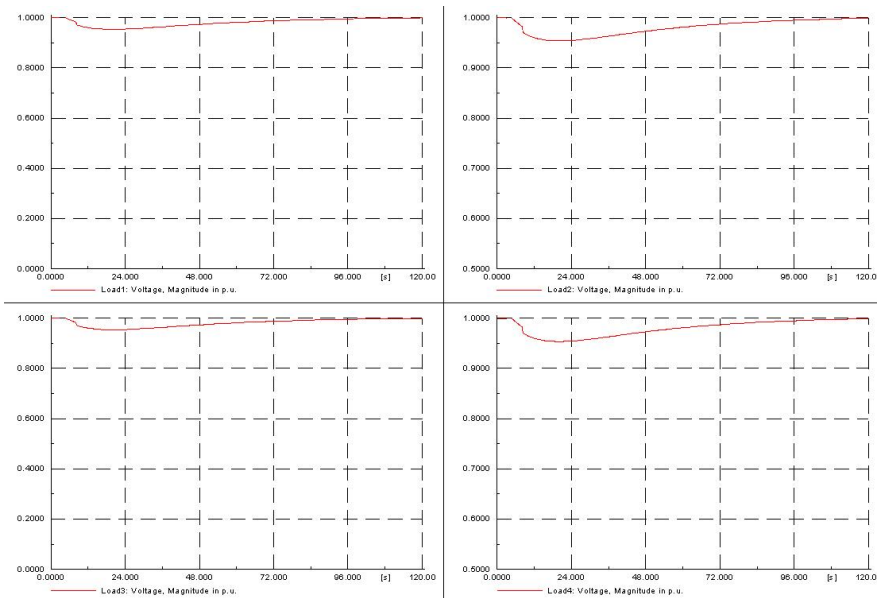

Fig. 7. Voltage response for all four loads during case I

\section{Simulations}

In this paper we present results from three different scenarios which have been simulated according to different patterns of service availability in the network presented in figure 3 . In all three scenarios, during the simulation, a part of the distribution network is islanded due to an outage at utility grid. Initially the system have three DGs connected, four loads and a connection to utility grid. Systems is balanced and voltage at the nodes of all loads is 1 p.u. Load agents at each load are continuously monitoring voltage to response to any disturbance.

\section{A. case I: Only one DG providing regulation service}

In first scenario, only one DG ( DG2 in this case) is providing regulation service. Which means that it can provide extra active power if required for balancing in the islanded part of system (the subgrid). The control flow in the system goes as follows in this scenario:

- Outage occurs at utility grid and a part of distribution network is islanded

- An amount of power coming from grid is lost which creates an imbalance in the islanded part of network

- Load agents observe voltage drop at their nodes

- Load agents contact DF agent to look for any regulation service

- DF agents informs about regulation service currently provided by the DG2 agent and provides its reference

- Load agents request the DG2 agent for provision of service

- The DG2 agent accepts this request and provides the service by increasing its active power setpoint accordingly

- Voltage is restored at nodes of all loads

Figure 7 shows voltage at loads 1 to 4 (starting from top left and moving clockwise) during the simulation. It shows an initial steady voltage of 1 p.u, a voltage drop due to outage and restoration after the provision of regulation service from DG2. Figure 8 shows communication of agents during the simulation of this case. For simplicity, only load agent1 has been shown in the communication. It can been seen that first load agent sends

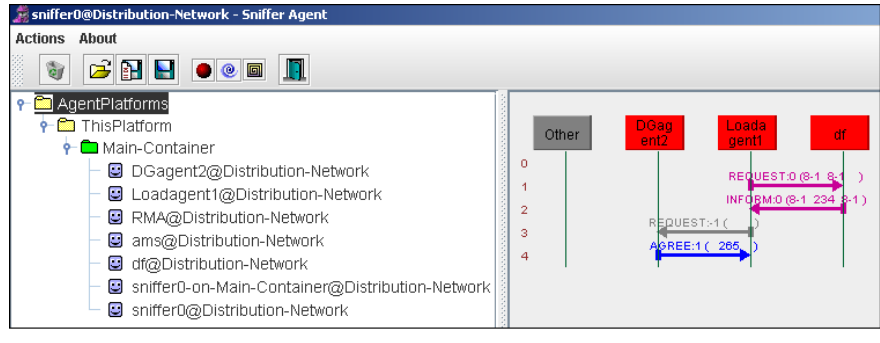

Fig. 8. Communication between agent during case I
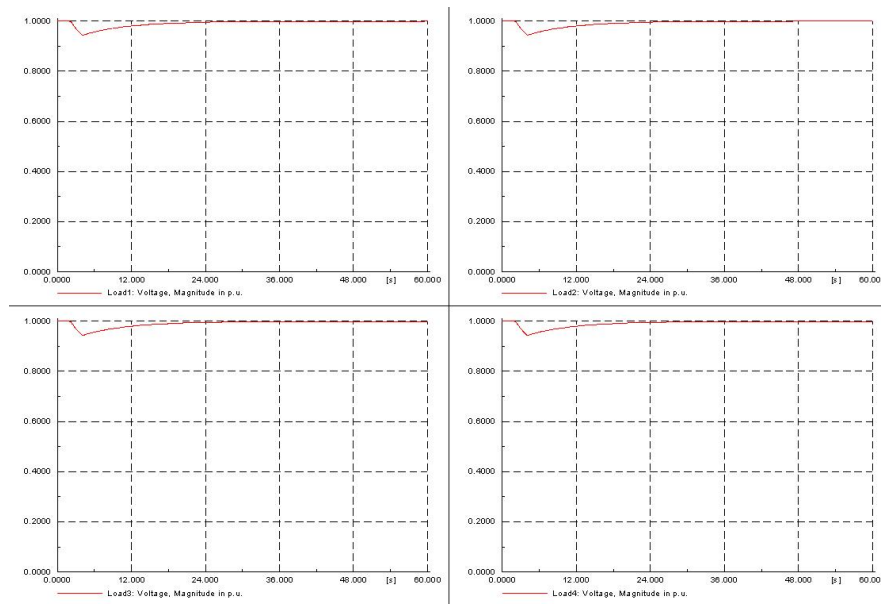

Fig. 9. Voltage response for all four loads during case II

a "Request" message to DF agent asking for the information about any available regulation service. In reply, DF agent sends an "Inform" message with information about available service and provider reference of this service. Load agent then sends a "Request" message to DG agent for provision of this service and DG agent replies with an "Accept" message.

\section{B. Case II: All three DGs providing regulation service}

In this case, all three DGs in the islanded part of distribution network are providing regulation service and subsequently accept the request from Load agent for provision of this service. Figure 9 shows the voltage response during the simulation of this case. It can be observed that voltage restoration is efficient in this case because regulation has been shared by three DGs. Figure 10 shows communication between agents during this case. It shows "Request" of load agent to all three DG agents which are currently providing regulation service and a subsequent "Accept" response from DGs

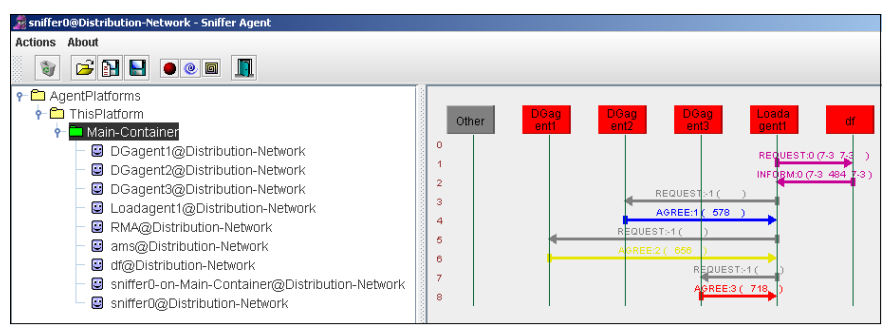

Fig. 10. Communication between agent during case II 


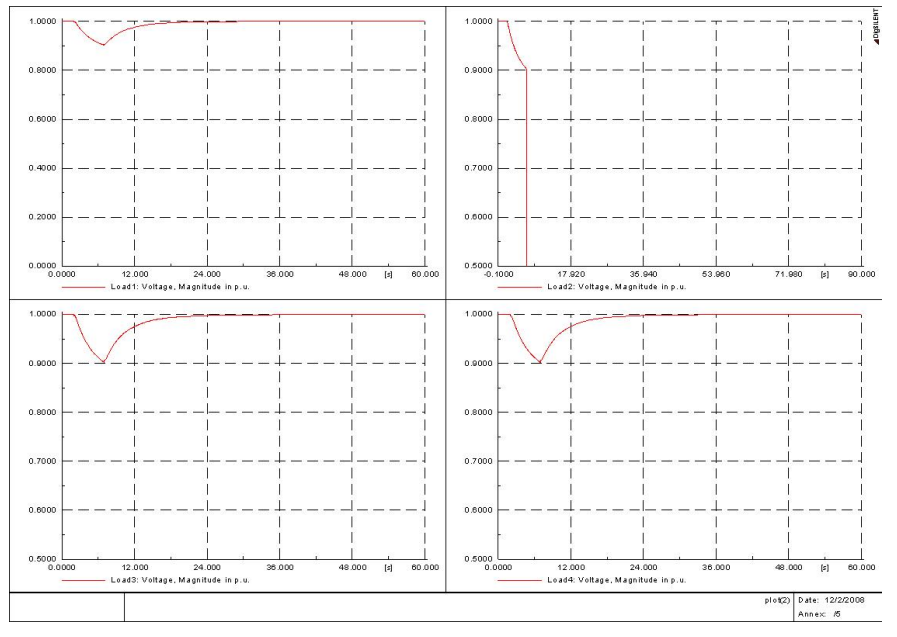

Fig. 11. Voltage response for all four loads during case III

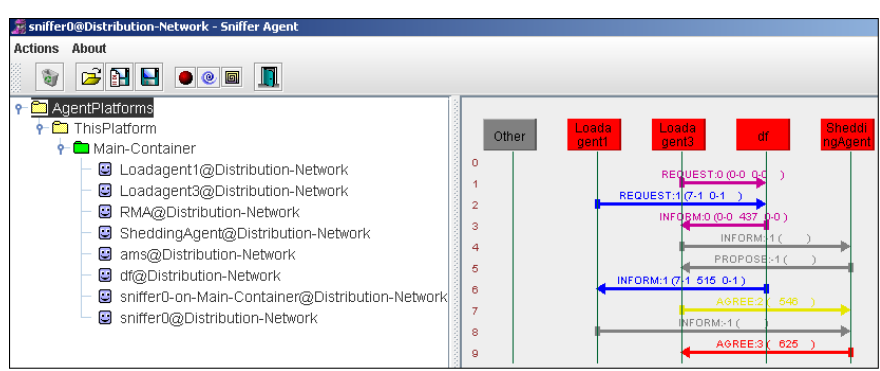

Fig. 12. Communication between agent during case III

\section{Case III: No regulation service available - Load Shedding}

This is the case where none of the DGs are providing regulation service at the time of outage when a part of distribution network is islanded from the utility grid. Load agents are informed about unavailability of any regulation service by the DF agent. Load agents inform this situation to the LoadShedding agent. LoadShedding agent proposes one of the the load agents, Load agent 3 in this case, to shed itself in order to achieve balance in the island. Load agent 3 agrees on this proposal and sheds itself. Voltage is restored as a result. Figure 11 shows the voltage response during simulation in this case. It shows an initial voltage drop due to outage and restoration after the load3 agent sheds itself. As a result, voltage goes down to zero at node of load3 and is restored at rest of the loads. Fig.12 shows communication between agents in this case. It can been seen that load agents send an "Inform" message to LoadShedding agent in order to inform it about situation of unavailability of any regulation service. LoadShedding agent then sends a "proposal" message to load3 agent which replies with an "Agree" response.

\section{Situation Awareness in Agents}

In experiments presented in previous section and in most of the work found in literature about using agents for developing industrial control systems, agents are assumed to be situation aware i.e. they have capability to respond adequately to inputs from the physical systems and from other agents. Two problems must be addressed here:
- How to define the situation or context of an agent's control actions?

- How is the agent informed about the situation?

In the following part of this section we present our ongoing work on how to make agents situation aware particularly in the context of control of distributed power systems and with reference to the case studies presented in previous section.

\section{A. Defining awareness in control situations}

When an agent delivers a control service, such as provision of balancing power, the action is always a part of context or situation. The action can be part of a plan that the agent has devised in order to accomplish its own goal or it can be seen as the agent's contribution to a community of several agents cooperating to achieve a common goal. As an example we consider the situation of failure of an action i.e. when an action of the agent is failed to produce intended results. In this case, it would be desirable that the agent could:

- Reconsider the objectives of the action and the means used

- Derive possible remedial actions and predict their consequences

- Plan and execute a (new) action

The agent may not make these decisions based on local knowledge alone. It may also be necessary to consider the global situation including knowledge about the role played by the agent as member of a community of agents and the purposes and functions of the physical power system components and subsystems. By getting situation aware, a requesting agent can ensure that services it requests will solve it's problem, whereas agent delivering services can ensure that the services it is delivering are dependable and that the requestor can rely on them.

Awareness about control situations can be ensured if the agent has an internal model representing the context of its actions. Ideally, the agent should not only have a library of behaviors but should also have knowledge base representing contextual knowledge required for handling abnormal situations. Such a knowledge base representing information about the control situation in a power system can be developed using multilevel flow modeling (MFM) [7]-[9]. The advantages of MFM is the ability to choose level of abstraction in the model so that it matches the particular need or perspective of each agent and that relations between perspectives are logically defined. In this way it can be ensured that the perspectives of each agent are consistent.

Figure 13 presents three views on the control situation in the example cases, presented in previous section, using the concepts of the MFM language for modeling means-end relations in complex systems. Three views are: systems's view, the view of one of the DG agents (DG3 agent) and a load agent (Load4 agent). Views for the other generators and loads are not included for simplicity of the presentation in figure 13 .

1) System's view - overall balancing: The part of the model comprising G1, $F_{S C H 1}, \mathrm{G} 2$ and $F_{S C H 2}$ represents the view of system - the overall balancing. This is a view of regulation of grid resources. Grid resources comprises three 
distributed generators represented by MFM source functions $S o_{D G 1}, S o_{D G 2}$ and $S o_{D G 3}$ and four loads represented by MFM sink functions $S i_{L 1}, S i_{L 2}, S i_{L 3}$ and $S i_{L 4}$. Furthermore the storage function labeled St represents the total rotating inertia in the system. The functions included in flow structure represent accordingly the resources involved in the balancing of power in the example case. The transfer of power from the generators to the loads is represented in MFM by the transport functions $\operatorname{Tr}_{D G 1}, \operatorname{Tr}_{D G 2}, \operatorname{Tr}_{D G 3}, \operatorname{Tr}_{L 1}, \operatorname{Tr}_{L 2}, \operatorname{Tr}_{L 3}, T r_{L 4}$. Since the control strategy adopted is decentralized so this view gets realized by the individual actions of agents.

2) The view of DGs: The view of DG3 is representing how the generator agent may see the control situation. From the perspective of the system, DG3 is simply a power source $S_{D G 3}$. But from the perspective of the generator agent, the grid is a power consumer or sink represented by $S i_{D G 3}$ and the power source feeding the generator is $S o_{D G 3}^{1}$. The inertia of generator DG3 is represented by an energy storage function $S t_{D G 3}$. The goal to be achieved by the generator agent is represented by $G_{D G 3}$. The goal specifies the power to be delivered to the grid.

3) The view of L4: The view of Load4 is representing how the load agent may see the control situation. From the perspective of the system, Load4 is simply a power consumer or load $S i_{L 4}$. But from the perspective of the load agent, the grid is a power source represented by $S o_{L 4}$ and the power consumer is represented by $S i_{L 4}^{1}$. Note that $S i_{L 4}$ in $F_{S C H 1}$ is not the same as $S i_{L 4}^{1}$ in $F_{L 4}$. The conversion of the power in the load from the electric energy e.g. to another form of energy is represented by the conversion function $C n_{L 4}$.

4) Relations between the three views: The relations between the views are indicated above. However, the MFM language allows systematic expansion and aggregation of functions so that e.g. the system's view may be expanded by incorporating the views of DG3 and/or Load4. In a service oriented agent architecture, this expansion could be done either as a demand from the system or could be done by the DG3 and Load4 agents explaining how they see the situation.

In this way the MFM models can be used to realize distributed situation awareness. This concept may appear conflicting with the basic agent paradigm which assumes that the agent is autonomous and takes actions based on local information only. But in reality MFM gives only a model of the world and not complete information. An agent, by using its local information, can perform reasoning on this model in order to decide e.g. which service it requires in current situation(from the load's perspective) or how to deliver a service so that it incorporates all dependabilities (from DG's perspective). An introduction to MFM and its application to power system is discussed in detail in [10] by current authors.

\section{CONCLUSIONS AND Discussion}

A dynamic software platform is developed to implement autonomous and local control of electric power systems with high penetration of DGs. A middleware software is implemented for real time communication between JAVA and DigSILENT power Factory simulation software. Results have

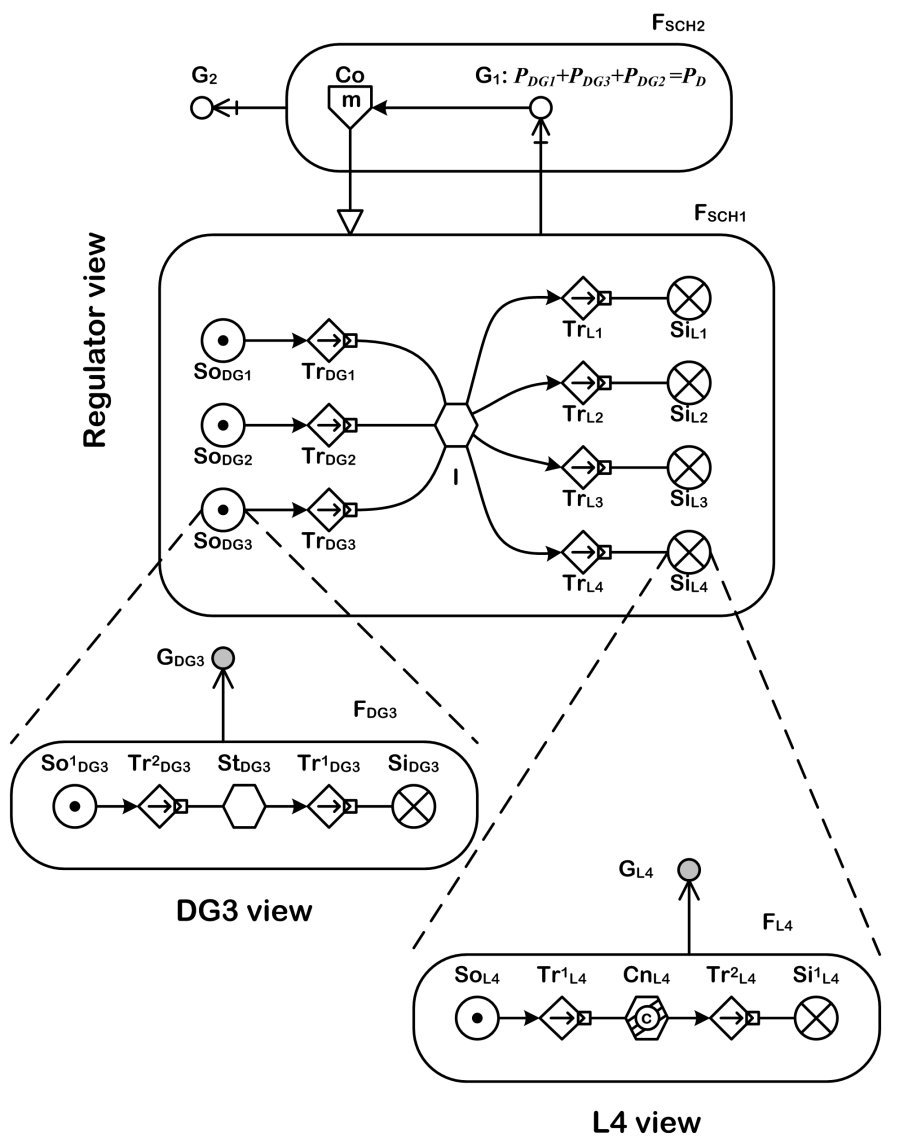

Fig. 13. Three views of the control situations

been presented from experiments of using this system in islanding operation of the low voltage distribution grid. It has also been described that how situation awareness is critical for agents to understand and reason about their environment specially in the action failure scenarios. A mechanism has been demonstrated for bringing situation awareness in agents during control situations. The work done and the results demonstrate that a careful use of agent technology and the service oriented architecture have great potential for designing control systems for the power system with significant amount of distributed generations. Also, increased autonomy in control systems requires that autonomous components should be able to grasp and understand the semantics of emerging control situations.

\section{REFERENCES}

[1] N. Jennings and S. Bussmann, "Agent-based control systems: Why are they suited to engineering complex systems?" Control Systems Magazine, IEEE, vol. 23, no. 3, pp. 61-73, June 2003.

[2] T. Nagata, Y. Tao, K. Kimura, H. Sasaki, and H. Fujita, "A multi-agent approach to distribution system restoration," vol. 2, July 2004, pp. II333-II-336 vol.2.

[3] S. McArthur, E. Davidson, and V. Catterson, "Building multi-agent systems for power engineering applications," Power Engineering Society General Meeting, 2006. IEEE, pp. 7 pp.-, June 2006.

[4] J. Solanki and N. Schulz, "Multi-agent system for islanded operation of distribution systems," 29 2006-Nov. 1 2006, pp. 1735-1740.

[5] M. Lind, T. Ackermann, P. Bach, H. W. Bindner, Y. Chen, R. GarciaValle, M. Gordon, K. Heussen, P. Nyeng, A. Saleem, P. E. Srensen, M. Togeby, I. Vlachogiannis, S. You, and Z. Xu, "Ecogrid: $50 \%$ wind power in the danish electric power system: System architecture wp-2 (phase 1)," 2008 
[6] Z. Xu, M. Gordon, M. Lind, and J. Ostergaard, "Towards a danish power system with $50 \%$ wind - smart grids activities in denmark," in Proceedings of the IEEE PES General Meeting, 2009, (invited panel paper, submitted).

[7] M. Lind, "The what, why and how of functional modelling," in Proceedings of International Symposium on Symbiotic Nuclear Power Systems for the 21'st Century (ISSNP), Tsuruga, Japan, July 9-11 2007, pp. 174179.

[8] - "Modeling goals and functions of complex industrial plant", Applied Artificial Intelligence, vol. 8, no. 2, pp. 259-283, 1994.

[9] - "Plant modeling for human supervisory control," Transactions of the Institute of Measurement and Control, vol. 21, no. 4-5, pp. 171-180, 1999.

[10] K. Heussen, A. Saleem, and M. Lind, "Control architecture of power systems: Modeling of purpose and functions," in Proceedings of the IEEE PES General Meeting, 2009, (submitted).

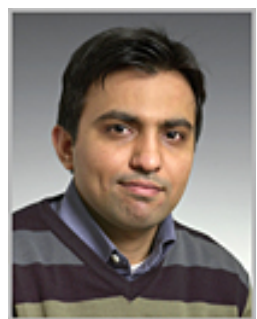

Arshad Saleem(M'07) is currently pursuing a Ph.D. degree from the Department of Electrical Engineering of Technical University of Denmark. He received his bachelors degree in computer science from University of the Punjab Lahore, Pakistan and an M.Sc degree in Artificial Intelligence from Blekinge Institute of Technology Karlskrona, Sweden in 2003 and 2007 respectively. His research interests are intelligent systems applications to power system, power system analysis and distributed control.

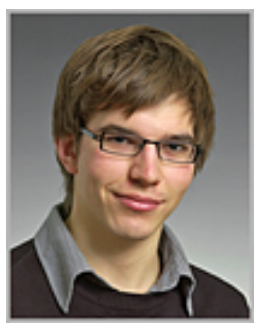

Kai Heussen(M'07) is currently a Ph.D. student in Automation and Electric Energy Systems at the Department of Electrical Engineering at the Technical University of Denmark. He studied control engineering in Stuttgart and Toronto and received his degree of Engineering Cybernetics from the University of Stuttgart in 2007. His research interests include control theory, distributed control architecture and energy sytems with very high shares of renewable energy.

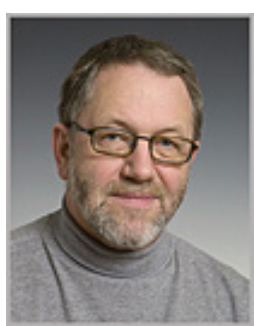

Morten Lind is Professor of Automation at Department of Electrical Engineering at Technical University of Denmark and is associated Centre for Electric Technology. His research interests include automation design, supervisory control of complex industrial systems and infrastructures, and application of agent technology and knowledge based system in automation. 